\title{
Figures de l'Estonie chez deux auteurs finlandais
}

Estonia seen by two Finnish authors

Eesti kahe Soome kirjaniku poolt vaadatuna

\section{Martin Carayol}

\section{(2) OpenEdition}

Journals

Édition électronique

URL : https://journals.openedition.org/efo/3569

DOI : $10.4000 /$ efo.3569

ISSN : 2275-1947

\section{Éditeur}

INALCO

\section{Édition imprimée}

Date de publication : 1 janvier 2014

ISBN : 978-2-343-05394-3

ISSN : 0071-2051

\section{Référence électronique}

Martin Carayol, «Figures de l'Estonie chez deux auteurs finlandais », Études finno-ougriennes [En ligne], 46 | 2014, mis en ligne le 09 octobre 2015, consulté le 08 juillet 2021. URL : http:// journals.openedition.org/efo/3569; DOI : https://doi.org/10.4000/efo.3569

Ce document a été généré automatiquement le 8 juillet 2021.

\section{(c) (1) (8)}

Études finno-ougriennes est mis à disposition selon les termes de la Licence Creative Commons Attribution - Pas d'Utilisation Commerciale 4.0 International. 


\title{
Figures de l'Estonie chez deux auteurs finlandais
}

\author{
Estonia seen by two Finnish authors \\ Eesti kahe Soome kirjaniku poolt vaadatuna
}

Martin Carayol

1 Cet article s'intéresse aux textes de deux auteurs, Aino Kallas (1878-1956) et Sofi Oksanen (1977-), qui ont fait des contrastes (réels ou imaginaires) entre Finlande et Estonie la substance même de certaines de leurs œuvres. La première a écrit les œuvres qui nous intéressent dans les années 1910 à 1930, alors que Sofi Oksanen est un auteur contemporain.

2 Avant d'évoquer en détail - en nous intéressant notamment à la figure du narrateur dans les textes considérés - la façon dont les deux auteurs dépeignent l'Estonie, il nous paraît utile de commencer par une brève présentation du contexte historique dans lequel s'inscrivent Kallas et Oksanen, en nous concentrant sur les relations culturelles entre Finlande et Estonie. ${ }^{1}$

\section{La culture, trait d'union historique entre la Finlande et l'Estonie}

3 L'intérêt finlandais pour les Estoniens est né dans la seconde moitié du XIX siècle, au moment de l'éveil national : cet intérêt était avant tout linguistique et culturel, mais il ne tarda pas à se politiser. L'amitié entre le fennomane Yrjö Koskinen ${ }^{2}$ et Johann Voldemar Jannsen ${ }^{3}$, à partir de 1864, peut être vue comme un symbole de ces premiers échanges culturels modernes. En 1869, quand Jannsen organisa les premières fêtes du chant en Estonie, des chants finlandais figuraient également au programme. Koskinen n'osa pas y aller par peur de représailles russes, mais il envoya le pédagogue C.G. Swan ${ }^{4}$, très enthousiaste, qui s'y rendit même plusieurs fois. Des fêtes du chant finlandaises furent ensuite organisées à Jyväskylä en 1884. 
4 En 1881 fut fondée la première association estonienne en Finlande, les Amis de la langue estonienne (Vironkielen ystävät). Koskinen encourageait Jannsen, mais Swan par exemple, dans le cadre de cette association, soutenait plutôt Carl Robert Jakobson ${ }^{5}$, qui était plus radical, et tenait pour une coopération plus étroite entre les deux pays. Swan et ses compagnons sont cependant assaillis de doutes après l'assassinat d'Alexandre II, par crainte de la russification : l'association est dissoute en 1883 à cause de ces dissensions.

5 Vers 1900, beaucoup d'étudiants estoniens se rendirent à Helsinki à cause de la russification (entamée par les autorités russes en 1885 par crainte d'une germanisation de l'Estonie ${ }^{6}$ ), mais ils n'étaient pas toujours appréciés car beaucoup s'engageaient dans la police ; cependant, beaucoup de mariages mixtes eurent lieu. Les Estoniens d'Helsinki créèrent en 1903 la Société estonienne de bienfaisance d'Helsinki (Helsingi Eesti Heategewa Selts), a priori destinée aux Estoniens, mais dans laquelle on trouvait en fait beaucoup de Finlandais intéressés par l'Estonie. Après la révolution de 1905, cette association fit figure de centre pour réfugiés; elle se politisa, et fut donc interdite en 1909 lorsque la répression s'intensifia.

6 En 1906 fut fondée l'Union finno-estonienne (Suomalais-Virolainen Liitto), créée par le poète estonien Gustav Suits et le folkloriste finlandais Kaarle Krohn : on y retrouve entre autres les écrivains Friedebert Tuglas et Hella Murrik (future Wuolijoki). Le nom de cette association pouvait inquiéter les autorités, mais elle était censée être purement universitaire, et ne posa donc pas de problème, jusqu'à ce que les Suédois se mettent à en parler dans la presse. C'est à ce moment-là que les autorités russes s'inquiétèrent ; elles y mirent fin en 1911.

7 À l'hiver 1918-1919, on assista en Finlande à un vif intérêt pour l'Estonie, beaucoup d'événements furent organisés, et l'on fonda le Comité général d'aide à l'Estonie (Viron Avustamisen Päätoimikunta), qui envoyait des volontaires combattre pour l'indépendance estonienne (car on ne souhaitait pas envoyer l'armée finlandaise à l'étranger) ; cette action s'interrompit en 1919 une fois le travail achevé et les Russes expulsés.

8 L'idéal de rapprochement des peuples apparentés (heimoaate), c'est-à-dire des peuples finno-ougriens, devint à la mode, les relations étaient aidées par l'indépendance de chacun des deux pays. On ne forma pas de nouvelles sociétés, mais celles qui existaient déjà fonctionnèrent à plein, comme Helsingi Eesti Selts. Professeurs et élèves participèrent à des programmes d'échange.

9 En 1921 fut fondé, notamment par Lauri Kettunen, professeur de langues fenniques à Tartu, le Club étudiant finno-estonien (Virolais-suomalainen ylioppilasklubi), dont le modèle était son équivalent de Tartu, le Club étudiant estonien-finnois (Eesti-Soome üliõpilasklubi). Les deux clubs se marièrent en 1923, formant le Club universitaire des peuples apparentés (Akateeminen Heimoklubi), très actif mais étonnamment peu politisé.

10 L'Union pour l'identité finlandaise (Suomalaisuuden Liitto), fondée en 1906, devint très importante dans les années 1920: en pratique, elle était concentrée sur l'Estonie, puisque la Hongrie et les peuples finno-ougriens de Russie étaient loin ou inaccessibles. La Société universitaire carélienne (Akateeminen Karjala-Seura soit AKS, avec notamment Urho Kekkonen, futur président finlandais), y acquit une grande influence en 1927, concentra encore davantage l'activité sur l'Estonie, avec notamment des aides 
au voyage. AKS possédait des bureaux à Helsinki et Tallinn, se servait de la radio pour donner des cours d'estonien, ce qui était une idée très moderne. Elle constituait en quelque sorte l'équivalent du Akateeminen Heimoklubi pour le grand public. En 40, les activités estoniennes s'arrêtèrent.

Les organisations étudiantes et AKS ont eu une grande importance dans les rapports finno-estoniens. Pour AKS, à l'époque où son meneur était Vilho Helanen (nationaliste, pionnier de la littérature policière en Finlande), il ne pouvait y avoir de Finlande indépendante sans Estonie indépendante.

En 1928, les corporations étudiantes d'Helsinki et de Tartu conclurent d'importants accords d'amitié.

\section{Tensions dans les années 30}

12 AKS devint peu à peu moins démocratique, certains soutinrent le mouvement de Lapua ${ }^{7}$ et la révolte de Mäntsälä8. Urho Kekkonen quitta la société, comme quelques autres. AKS soutint également la très populiste Ligue des vétérans de la guerre d'indépendance (Vabadussõjalaste Liit), les vapsid, équivalent estonien du mouvement de Lapua, mais avec un tout autre succès électoral ${ }^{9}$. En 1934, Konstantin Päts ${ }^{10}$ interdit la Ligue des vétérans; AKS et toute la droite finlandaise l'accusèrent d'être un dictateur. De ce fait, on constate beaucoup de tensions dans les rapports finno-estoniens: des étudiants finlandais invités en Estonie prononcèrent des discours contre Päts et furent par la suite chassés du pays.

13 Artur Sirk, meneur de la Ligue des vétérans, s'enfuit et se réfugia en Finlande, aidé par AKS. En 35, il tenta un contre-coup d'État en Estonie. En conséquence, beaucoup d'Estoniens changèrent de sentiment envers les Finlandais, supposés être anti-Päts. À l'enterrement de Sirk au Luxembourg en 1937, des membres d'AKS accusèrent l'Estonie de l'avoir assassiné: les relations étaient au plus bas, l'Estonie rappela son ambassadeur...

De ce fait, certains regrettèrent d'avoir mêlé la politique et l'activisme culturel ; tous les amoureux de l'Estonie furent catalogués à droite et considérés comme soutenant la Ligue des vétérans contre Päts. En 1936 fut fondée la Société finno-estonienne (Suomalais-Virolainen Seura soit SVS), financée par le gouvernement et dirigée à partir de 1938 par Urho Kekkonen, alors ministre de la justice. Même peu active, SVS permit de calmer les relations finno-estoniennes en se concentrant sur la culture.

Après la guerre d'hiver (à l'hiver 1939-1940), l'Estonie invita en été des enfants ayant souffert de la guerre : participaient à cette initiative l'Union pour l'identité finlandaise et SVS. Mais cette initiative ne dura pas longtemps, les Soviétiques arrivèrent et tous les Finlandais furent promptement rapatriés.

16 Avec l'occupation allemande, l'estophilie chuta, car on ne souhaitait pas en Finlande fâcher l'allié allemand. Les Finlandais gardèrent profil bas pendant la guerre. En 1943, le nombre de réfugiés estoniens augmenta et l'Allemagne exigea de la Finlande qu'elle lui livrât tous ces gens; SVS, à présent dirigée par Kekkonen, s'occupait au contraire de prendre soin des réfugiés, notamment au domaine de Jollas à Laajasalo.

17 Bref, des organisations privées se chargèrent, financées par l'État, de tâches qui auraient dû incomber à celui-ci; mais les dirigeants finlandais se voulaient très prudents. Lors de l'hiver 1943-1944, l'aide aux réfugiés (dont le nombre explosait) 
devint très visible, mais l'Allemagne avait désormais d'autres problèmes : on pouvait désormais aider les réfugiés presque sans se cacher! En août 1944, beaucoup de réfugiés partirent défendre leur patrie contre les Russes, mais le succès fut médiocre. Ils repartirent, mais pas en Finlande, ils continuèrent leur route vers la Suède car la Finlande était contrainte par ses accords avec la Russie. Commença alors une longue période de calme dans les relations finno-estoniennes.

\section{Pendant l'occupation soviétique de l'Estonie}

Avant 1955, le voyage en Estonie était presque impossible et, même après cette date, il demeura très compliqué. De toute façon, la haine envers l'Estonie soviétique était patente parmi les estophiles. Cela changea après la visite de Kekkonen en 1964 et avec la montée du socialisme : on accepta davantage l'appartenance de l'Estonie à l'URSS, mais il y eut évidemment beaucoup de débats et de conflits à ce sujet.

Toutes les «sociétés des peuples apparentés» (heimoseurat) durent arrêter leurs activités à l'hiver 1944-1945 pour ne pas fâcher la Russie. L'Union pour l'identité finlandaise parvint malgré tout à aider les proches de réfugiés rendus à l'URSS, élaborer un abécédaire en estonien distribué en Suède aux réfugiés, entre autres missions, mais elle aussi cessa ces activités vers 55 . De manière générale, on s'intéressait davantage en Finlande aux exilés qu'aux Estoniens restés en Estonie, ce qui se manifeste notamment dans le fait qu'on traduisit beaucoup de littérature exilée, mais pas beaucoup de littérature estonienne soviétique, jusqu'aux années 60. Ceci est également lié au fait qu'il y avait davantage de parutions estoniennes hors d'Estonie.

On trouve cependant un rare exemple d'enthousiasme aveugle pour l'URSS dans la Société Finlande-URSS (Suomi-Neuvostoliitto-Seura), fondée en 1944, avec une section estonienne fondée à partir de 1957 à l'époque de la déstalinisation, quand Hruščëv voulait réchauffer les liens avec l'Ouest. Ses membres voulaient pour la Finlande le même destin soviétique que l'Estonie. Par exemple, l'organisation culturelle soviétique VOKS (Société pour les relations culturelles avec l'étranger) se tourna vers l'Estonie et proposa de travailler en collaboration avec SNS. Parmi les personnes impliquées dans SNS figurent Raili Kilpi (un futur membre de la Société Tuglas, dont il sera question cidessous), et Kustaa Vilkuna (folkloriste, linguiste et homme de confiance de Kekkonen). En 1957 est organisée une exposition de littérature estonienne (soviétique) à Helsinki, puis une exposition de littérature finlandaise à Tallinn l'année suivante. Le but, pour les autorités soviétiques, était de faire de la propagande dirigée vers l'Ouest. Elles pensaient que les Finlandais pourraient être séduits par l'Estonie soviétique, qui se portait mieux que beaucoup d'autres pays d'URSS. En protestation, les estophiles (comme Lauri Kettunen) organisèrent une exposition de littérature estonienne en exil.

21 Il existe une troisième voie, entre rejet et admiration pour l'Estonie soviétique, voie représentée par Eeva Niinivaara: elle était intéressée par toute la littérature estonienne, d'exil ou soviétique, et jamais ne prit position publiquement contre l'Estonie soviétique, même s'il est clair qu'elle ne la voyait pas d'un très bon œil (notamment parce que certains de ses proches avaient été envoyés en Sibérie). Elle fut très active comme lectrice à Helsinki de 1945 à 1969 et organisa beaucoup d'événements culturels. Elle avait beaucoup de liens avec les Estoniens de l'exil, bien sûr, mais s'en forgea également avec des Estoniens restés au pays, au risque de perdre des amis qui lui reprochaient cette ouverture vers l'URSS. Se retrouvaient chez elle tant 
des Estoniens exilés que des Estoniens soviétiques, en même temps parfois. Elle réussit à inspirer à beaucoup de ses étudiants l'envie de privilégier la culture sur la politique et donc de se montrer conciliants envers l'Estonie soviétique. Elle envoya beaucoup d'étudiants travailler leur estonien avec des Estoniens de l'exil, dans des camps d'été en Suède. Cette dernière initiative s'arrêta à la suite de la visite de Kekkonen en Estonie, qui imposa un arrêt des échanges entre Finlandais et Estoniens de l'exil.

Kekkonen avait longtemps voulu se rendre en Estonie après la guerre, mais ce n'est qu'en 1964 qu'il en eut l'autorisation, dans le cadre d'une visite non officielle. Beaucoup d'estophiles se lamentèrent, car cela revenait à donner un blanc-seing à l'occupant soviétique; les Occidentaux s'inquiétèrent également. En Estonie au contraire, on se réjouit, surtout parce que Kekkonen utilisa toujours l'estonien lors de sa visite. De manière générale, il se montra estophile lui-même, parlant de la parenté finnoougrienne, de l'importance de la culture estonienne ainsi que de son ami Friedebert Tuglas, bien que celui-ci fût à l'époque mal vu des autorités. Il souhaita que se ravivent les relations finno-estoniennes : pour beaucoup d'Estoniens, ce fut la preuve que leur pays n'était pas complètement oublié. Il se prononça pour plus d'échanges culturels, ce qui eut pour résultat la remise en marche des lignes navales HelsinkiTallinn qui avaient été interrompues depuis la guerre d'hiver.

On peut se demander pourquoi les Soviétiques acceptèrent la reprise des échanges culturels avec la Finlande. Ils espéraient sans doute rendre l'attitude des Finlandais envers l'Estonie soviétique plus positive, et le tourisme était bien sûr aussi un moyen d'attirer des devises. Visiblement, en tout cas, ils ne comprirent pas les conséquences de la reprise des échanges. Les échanges culturels, les relations d'amitié se développèrent de façon considérable et tout le monde s'accorde à penser que cela rendit l'Estonie moins totalitaire. Bref, cette visite de Kekkonen eut une influence très bénéfique.

Cependant, il y avait une condition : les Finlandais devaient cesser tous rapports avec les Estoniens de l'exil, ce que Kekkonen expliqua très clairement à ses amis estophiles. Et en effet, les relations avec les exilés s'effondrèrent, la presse finlandaise favorisa majoritairement la nouvelle orientation. Les étudiants d'Helsinki arrêtèrent d'alimenter les fonds servant à conserver des liens avec les réfugiés. Eeva Niinivaara arrêta d'envoyer des étudiants en Suède apprendre l'estonien et les traductions de livres publiés en exil cessèrent complètement.

Une exception notable est le livre 20 nykyvirolaista runoilijaa (Vingt poètes estoniens contemporains, dir. Raili Kilpi et Arvo Turtiainen, 1969), où figuraient une moitié d'écrivains exilés. C'était une sorte de réaction à la politique de Kekkonen. De même, SKS ${ }^{11}$, bastion de l'estophilie, organisa en 1967 une exposition de littérature estonienne en exil. Kekkonen protesta par l'entremise de son homme à tout faire dans le domaine de la culture, Kustaa Vilkuna, et par la suite SKS se concentra, en tout cas publiquement, sur l'Estonie soviétique, se soumettant ainsi à la ligne Kekkonen.

Par la suite, les preuves de la santé retrouvée des relations finno-estoniennes abondent. En 1967, on ne compte pas moins de dix-neuf compétitions sportives finno-estoniennes. La même année, on trouve à Oulu, pour la première fois, un lecteur d'estonien venu d'Estonie soviétique, puis un autre l'année suivante à Helsinki. Les échanges scientifiques s'accélèrent. C'est un beau succès en somme. On peut aussi évoquer ici les congrès finno-ougristes, en 1965 à Helsinki avec la participation de beaucoup 
d'Estoniens, en 1970 à Tallinn avec beaucoup de Finlandais... Le sommet des échanges culturels est atteint en 1969 au festival de chant de Tallinn.

Dans tout cela, SNS eut un rôle crucial, plus encore que le ministère de l'Éducation. SNS recréa pour l'occasion une section estonienne, dirigée par Lauri Posti, finno-ougriste et ami (au même titre que Vilkuna) de Kekkonen. Toute personne souhaitant organiser une manifestation en rapport avec l'Estonie devait prendre contact avec SNS, ce qui rendait toute initiative facilement contrôlable...

Il y eut un refroidissement entre 1968 et le début des années 1970, après le printemps de Prague, les Soviétiques rentrant dans leur coquille. Les autorités faisaient des difficultés pour envoyer des lecteurs d'estonien, les compétitions sportives finnoestoniennes prirent fin. Moscou commença à se demander pourquoi l'Estonie était le seul pays soviétique à avoir une section dédiée chez SNS. Les Soviétiques entreprirent donc d'espionner les activités de SNS, des communistes sûrs surveillèrent les réunions. Par conséquent, SNS s'affaiblit, très peu de ses projets purent désormais se réaliser ; la frustration des personnalités culturelles était immense et Lauri Posti partit en 1973. Les partisans d'une collaboration rapprochée avaient un ennemi juré, Toivo Karvonen, le secrétaire du Parti communiste, qui bloquait tout.

Toutefois, la situation n'avait rien de comparable avec ce qui s'était passé avant 1964 ; d'ailleurs, les voyages des Finlandais vers l'Estonie se multipliaient. Les Estoniens en revanche ne pouvaient guère quitter l'Estonie. Il n'en demeure pas moins que, de tous les Soviétiques, ils étaient ceux qui connaissaient le mieux la vie à l'occidentale, ayant notamment accès à la télévision finlandaise.

Les rares conversations téléphoniques outre-golfe étaient surveillées, ainsi que la correspondance: en général, les voyageurs se proposaient d'amener eux-mêmes des lettres d'amis, pour éviter les surveillances politiques. Les guides touristiques (comme Eva Lille, également importante traductrice) étaient surveillés car soupçonnés de s'adonner à la contrebande.

31 Dans les années 1980, l'URSS devint militairement plus agressive, et chercha à se protéger intérieurement en russifiant à l'ouest. En 1978 fut conclu un accord secret sur la russification des Estoniens et le secrétaire du Parti estonien devint Karl Vaino, qui parlait à peine estonien. Par l'intermédiaire de la Finlande, l'Occident fut informé, ce qui ralentit un peu le mouvement de russification. Et à partir de 1980, les Estoniens apprirent ce qui se passait en Pologne, se dirent qu'il était possible de résister et qu'il n'y avait rien à perdre. C'est à cette époque qu'eut lieu l'épisode de la «lettre des quarante ", lettre ouverte des intellectuels (y compris des membres du Parti) sous la direction de Jaan Kaplinski ; Moscou donna l'ordre à Tallinn de régler sans bruit cette agitation naissante. Les autorités estoniennes firent peur aux signataires, leur interdirent de voyager, etc. L'épisode montra clairement qu'il était devenu envisageable de critiquer le régime. ${ }^{12}$

\section{La Société Tuglas (Tuglas-seura)}

En Finlande, la mode communiste passa à la fin des années 70, et dans le même temps les Soviétiques commencèrent à exercer moins de pression sur la politique finlandaise. L'autocensure disparut peu à peu, surtout après le départ de Kekkonen en 1981. Entre 1978 et 1981, on s'intéressa beaucoup dans les médias finlandais à la politique de russification en Estonie. Les personnes désireuses de fonder une association d'amitié 
finno-estonienne étaient toutefois renvoyées à Suomi-Neuvostoliitto-Seura, dont elles ne voulaient plus à cause de son soviétisme. C'est dans ce contexte que naquit, lors d'une fête organisée en l'honneur d'Eeva Niinivaara, l'idée de la Société Tuglas. L'accord de paix interdisait les associations d'amitié existant avant la guerre mais ne semblait pas interdire d'en créer de nouvelles.

La Société s'orienta vers la culture et en particulier la littérature, car la fondation d'une société d'amitié, ou plus politique, semblait périlleuse; dans les statuts, on ne se prononçait pas sur la nature politique de l'Estonie, pays soviétique nageant dans le bonheur ou pays occupé par les Soviétiques. On peut lire dans ce positionnement l'influence de Niinivaara, son goût du compromis et du juste milieu.

Le choix du nom est également révélateur: Friedebert Tuglas est un écrivain non soviétique, non exilé, d'avant 1940, mais bien vu des Soviétiques (autrement la coopération avec l'Estonie contemporaine eût été impossible) après une longue période, notamment sous Staline, de mise à l'index, et, de plus, Tuglas avait des liens étroits avec la Finlande. La société est fondée en 1982, en présence de membres de SKS et de la section estonienne de SNS. La direction élue était clairement orientée vers la littérature, avec des traducteurs, des chercheurs et des éditeurs, entre autres.

Pour Moscou, la Société Tuglas était acceptable, car elle dispensait les autorités de devoir contrôler des individus isolés (les estophiles refusant par ailleurs le cadre fourni par SNS) : il est toujours plus facile de surveiller une organisation. Curieusement, les autorités soviétiques d'Estonie, avec Karl Vaino et Rein Ristlaan à leur tête, étaient plus méfiantes. Mais sous Gorbačëv, leur position devint difficilement tenable.

La Société Tuglas fit beaucoup de publicité et son succès fut assez rapide, avec la visite du grand écrivain Jaan Kross à Helsinki par exemple, transmettant les salutations de l'Union estonienne des écrivains.

En novembre 1982, la soirée poétique "Tuulen lapset " remporta un grand succès, beaucoup de gens voulaient montrer leur soutien à l'Estonie souffrant alors des pressions russifiantes. Parmi les invités, les poètes Juhan Viiding et Paul-Eerik Rummo faisaient partie des signataires de la « lettre des quarante » et étaient donc interdits de voyage; toutefois, Rummo obtint finalement l'autorisation, car les Soviétiques craignaient pour leur image en Finlande.

Le but de la société Tuglas était de favoriser l'organisation de manifestations estophiles, et non pas nécessairement de s'afficher comme leur organisateur; souvent, c'étaient d'autres structures plus riches qui se chargeaient de l'organisation officielle.

39 Dans les années 1983-1986, l'Estonie jouit d'une grande popularité dans les médias finlandais, et ce en grande partie grâce à la Société Tuglas. Ainsi, le centenaire de Tuglas en 86 fut un événement important.

En 1987 et 1988 a lieu l'effondrement rapide du pouvoir communiste; dans le même temps, en Estonie, le Rahvarinne d'Edgar Savisaar appuie les innovations de Gorbačëv. Le 16 novembre 1988, le soviet suprême d'Estonie se désolidarise de l'Union soviétique. Mais le Parti n'arrive pas pour autant à contenter le désir de liberté, et il disparaît ; Savisaar devient premier ministre. Dans le même temps, en Finlande, on assiste à beaucoup de changements dans la Société Tuglas: les programmes culturels se réalisent beaucoup plus facilement et l'on se détourne de SNS dont on avait tant besoin par le passé ; le poids de la société est accru par l'intérêt considérable des médias pour les événements en Estonie. 
41 En 1988, le ministère finlandais de l'Éducation, avec plusieurs estophiles vigoureux à sa tête, décide de bâtir des liens très étroits avec l'Estonie et propose de financer la Société Tuglas. Kalervo Siikala, responsable de ces mesures, n'était pas ministre mais a pris des décisions de grande importance pour le ministère. Il a rallié la ministre à son estophilie et, derrière ces manœuvres, le président Koivisto lui-même donnait son aval. La ministre Anna-Liisa Kasurinen était présente à certaines manifestations de la Société Tuglas, pour voir de ses propres yeux si les activités n'étaient pas trop politiques.

Parmi les activités culturelles des années 1987-1991, la plus importante est en 1989 la réunion des écrivains d'exil et des écrivains d'Estonie soviétique. Au début, les exilés sont farouchement contre, mais les gens de la Société Tuglas se montrent habiles, choisissent les bons interlocuteurs. L'événement est important car par la suite l'Estonie a commencé à publier des exilés; il est resté des méfiances et des rancunes entre les deux groupes, mais de manière générale la réconciliation a eu lieu.

En 1990-1991, la Société Tuglas ne se contente plus de favoriser la culture estonienne, elle favorise également les agents de l'indépendance estonienne, en priorité Lennart Meri.

\section{L'Estonie dans l'œuvre d'Aino Kallas}

Aino Kallas est née Aino Krohn, elle est la fille du grand folkloriste finlandais Julius Krohn ; en 1900, elle épouse Oskar Kallas, un Estonien, diplomate, folkloriste et linguiste, et le suit dans ses déplacements, en Russie et en Estonie, ne revenant plus en Finlande que pour de courtes périodes.

\section{Le journal intime d'Aino Kallas}

\section{Un sentiment de déracinement}

On sent bien à la lecture du journal d'Aino Kallas à quel point la période qui suit son mariage avec Oskar Kallas a été difficile, du point de vue des relations sociales. À plusieurs reprises elle se dit notamment déçue par la compagnie des Estoniens et, surtout, des Estoniennes. Ainsi le 4 octobre 1900 :

Une fois j'étais dans une nombreuse compagnie, mais j'en suis partie la gorge presque serrée par les pleurs, tant je m'étais ennuyée. Les femmes sont toutes mesquines, ne s'intéressant à rien, enfermées dans leurs étroites conceptions. Les hommes, au moins, sont patriotes, ils sont attachés à leur langue et à leur nationalité, alors que les femmes ne s'en soucient nullement. Les domestiques et l'économie du foyer, les intrigues familiales - c'est là tout leur monde. ${ }^{13}$ (Kallas 1978, p. 243)

Ainsi, elle est très critique envers la famille de son beau-frère Rudolf Kallas, où, à cause de l'influence de la femme de ce dernier, c'est l'allemand qui est parlé à la maison et non l'estonien. Elle écrit, le 26 février 1902, que ses yeux, pendant l'hiver, se sont ouverts à beaucoup de choses, " notamment en ce qui concerne l'identité estonienne» :

L'estonitude est à un tournant, avec d'un côté la germanité qui constitue un Scylla, de l'autre le Charybde russe. Parviendra-t-elle à louvoyer? Oui, si les femmes se réveillent ! Ces femmes baltes, dont l'horizon de pensée est si étroit et borné, qui ne savent ni discuter, ni s'enthousiasmer, ni réfléchir ! [...] 
J'ai connu mon "réveil estonien " par une voie tout à fait négative, à travers la femme et les filles de Rudolf Kallas, car elles sont absolument froides et insensibles à l'estonité, elles vont jusqu'à traiter avec mépris toutes les revendications estoniennes. (Kallas 1978, p. 266) ${ }^{14}$

Elle est particulièrement indignée par le fait que les enfants parlent à peine estonien :

La cadette, Grete, une écolière belle, poétique, un peu superficielle :

- Qu'y puis-je, si j'aime les Allemands bien plus que les Estoniens? Le peuple estonien est si terriblement bête !

- Vraiment? Ton père aussi ?

- Oh, mais papa ne fait pas partie du peuple.

On ne peut lui arracher un mot d'estonien, elle répond en allemand avec entêtement, elle lit l'estonien à grand-peine et dit ne le comprendre que partiellement. [...]

Aksel, petit garçon de onze ans, n'a jamais, à l'en croire, tenu de livre estonien entre ses mains. Il a été grandement surpris d'apprendre que son père appartenait à la Société des étudiants estoniens [...] et a dit que lui-même souhaitait appartenir à une corporation allemande. (Kallas 1978, p. 267)

Elle exprime aussi clairement le sentiment d'être entre deux pays, de n'être plus finlandaise et de n'être pas tout à fait estonienne. Ainsi, dans une lettre à Gustav Suits, le 15 janvier 1908, en réponse à une critique de celui-ci quant à son choix de traiter des sujets estoniens :

Il y avait dans votre critique un passage où vous avez touché un point particulièrement sensible en moi. Je pense ici à mon «déracinement » et à ma relation à la compréhension ou à l'incompréhension des réalités estoniennes et de l'âme de ces réalités. ${ }^{15}$ (Kallas 1978, p. 405)

Elle explique que la vie en Estonie lui a ouvert les yeux sur la réalité, alors que la Finlande, avec ses contradictions et ses luttes internes, lui est devenue trop étrangère pour cela ${ }^{16}$.

50 Le passage le plus important de cette lettre à Suits est celui où Kallas explique qu'audelà du choix du sujet traité (sujet finlandais ou sujet estonien), l'essentiel pour elle est de parler de la véritable humanité et non de telle ou telle nation :

Mon erreur [...] est de vouloir traiter des problèmes estoniens. Ma vision intérieure n'y suffit évidemment pas. C'est une tâche qui incombe à des écrivains estoniens. Mais vous comprendrez bien la distinction, quand je vous dirai que flotte dans mon esprit une idée qui ressemble à ceci : traiter des problèmes humains généraux, mais avec des personnages estoniens. Peut-être cela n'est-il pas dit suffisamment clairement, mais pour le moment je ne sais pas le dire mieux. Car où irais-je chercher mes personnages à l'avenir - certainement pas en Finlande ! [...]

Si bien que si je pouvais choisir librement, je ne chercherais pas à avoir des racines en Finlande ni en Estonie, mais dans cette grande humanité, sur laquelle nous pouvons tous nous greffer et croître. (Kallas 1978, p. 408) ${ }^{17}$

51 En 1917, en pleine agitation politique, Aino Kallas affirme : «J'aime la Finlande, mais le destin de l'Estonie est plus cher à mon cœur. » (Kallas 1978, II, p. 27) ${ }^{18}$

Ce sentiment de se rapprocher intimement du destin de l'Estonie trouve son point d'orgue le 16 novembre 1918, au moment où l'Estonie devient indépendante :

Pendant quinze ans j'ai vécu en Estonie, pendant dix-huit ans j'ai côtoyé les conceptions de ce peuple, j'ai toujours été une étrangère, toujours à l'extérieur jusqu'à maintenant! [...] La Finlande, pour moi, s'est fragmentée en personnes individuelles, d'un point de vue collectif c'est maintenant l'Estonie qui est mon pays. ${ }^{19}$ (Kallas 1978, II, p. 114) 


\section{L'amour de la nature estonienne}

Aino Kallas se montre souvent enthousiaste, et ce dès ses premières années en Estonie quand elle décrit les paysages vus lors de ses excursions diverses, principalement sur les îles estoniennes. Ainsi, les 7 et 8 juillet 1907, sur la presqu'île de Sõrv à Saaremaa, elle écrit notamment : «[...] je ressentis soudain un souffle poétique en contemplant le vieux parc et la maison abandonnée ", ou encore "Le gardien du phare, à notre vue, allume la lanterne. C'est un spectacle d'une beauté féerique.» (Kallas 1978, p. 305 et 306) les bouleaux embaumaient, Sulev reposait sur mon sein, la pleine lune brillait à travers les saules-lauriers. Je me disais que j'avais un petit garçon si beau, si charmant. Je pensais à Saaremaa avec gratitude, parce que je sens ici - seulement ici - que j'ai trouvé mon foyer (Kallas 1978, p. 525) ${ }^{21}$. l'étroit », expression qui revient souvent. En 1916, son amant Jan Rosenthal lui demande : "Comment était Tartu? Trop étroit? - Trop étroit, comme d'habitude, répondis-je $»^{22}$ (Kallas 1978, p. 712).

Le 14 mai 1917, elle écrit à propos de Tartu :

Jamais encore la petitesse et l'étroitesse de cette ville ne m'avaient sauté aux yeux comme à présent (Kallas 1978, II, p. 53).

Alors que Pärnu l'enchante immédiatement ${ }^{23}$, Tartu lui demeure longtemps pénible et étrangère, même si les choses s'améliorent au fil des ans.

\section{Une terre vouée à Satan}

On connaît le dispositif narratif employé par Kallas dans ses nouvelles estoniennes : le narrateur y est en général un pasteur, un membre de la classe dominante germanobalte, aux vues conservatrices, et qui porte un regard critique tant sur la plupart des manifestations de l'identité estonienne que sur la conduite selon lui répréhensible des héroïnes dont il retrace l'histoire, Barbara von Tisenhusen, la femme du pasteur de Reigi (ce dernier étant le narrateur de sa propre histoire), Aalo la louve...

En corollaire de ce dispositif narratif, on peut identifier plusieurs topoï courants dans les nouvelles kallasiennes et, en premier lieu, la peinture d'une Estonie où règnent le mal et le péché.

Ainsi, dans «Barbara von Tisenhusen » :

Or en ces temps-là [...] s'accroissaient en Livonie l'aisance et la richesse, mais également toutes sortes de mauvaises herbes plantées par Satan, comme l'ostentation et la volupté tant parmi les gouvernants qu'au sein du petit peuple. Car les bourgeois ainsi que les paysans non-germaniques avaient imité l'exemple de leurs seigneurs, si bien qu'il n'existe aucun autre peuple dans toute la chrétienté qui ait vécu dans une telle débauche, à la manière des Sodomites et des Épicuriens, chacun cherchant son bonheur dans une joie terrestre et pécheresse. ${ }^{24}$ (Kallas 2008, p. 122) 


\section{de la tentation charnelle :}

\begin{abstract}
Mais quand j'arrivai à Tallinn, Estonie et Livonie avaient souffert d'une guerre incessante pendant près de soixante-dix ans, et tout le pays donnait l'impression que les étalons du Saint Livre des Révélations l'avaient foulé de leurs sabots. Les églises avaient été brûlées ou autrement profanées. [...] Et, bien souvent, les pasteurs n'avaient rien de pasteurs, si ce n'est leur cape noire. [...] Et Satan, ce Vieux Pêcheur des Enfers, marchait aux alentours et jetait ses filets dans l'eau trouble. Certes, des bûchers de sorcières flambaient partout dans le pays, brûlant chardons et mauvaises herbes, répandant une odeur nauséabonde, mais de nouveaux exaltés apparaissaient comme de la bouche des Enfers, car le Démon semblait avoir décidé que l'Estonie ne serait jamais bienheureuse. ${ }^{25}$ (Kallas 2008, p. 157)
\end{abstract}

60 Enfin, citons, de «La fiancée du loup », la plus célèbre nouvelle de Kallas, le passage suivant, où est décrite l'invasion de loups-garous, qui fait suite à une prolifération de loups «naturels»:

Mais ces hordes de loups téméraires n'étaient que les avant-gardes des Enfers, que l'Esprit Lubrique, toujours disposé à mener des humains à leur perte, envoyait devant lui. Car peu après que ces loups naturels se furent tant exaltés et déchaînés, au point qu'il était impossible de se mettre nulle part à l'abri de leurs tours, déjà des humains commencèrent à courir le garou et se livrer à des actions lupines, comme si un vil Démon les eût possédés. [...]

Et c'est ainsi que sur la Terre du Diable également, des brebis du troupeau du Seigneur tombèrent dans ce nouveau piège tendu par Satan. ${ }^{26}$ (Kallas 2008, p. 242-243)

\title{
Une terre de superstitions
}

61 L'autre topos que nous voudrions examiner ici est celui qui consiste pour le narrateur à flétrir la vitalité des traditions païennes en Estonie - position diamétralement opposée à celle de l'auteur. Citons encore une fois la nouvelle «Barbara von Tisenhusen » :

De même, elle avait commencé à passer son temps d'une façon frivole, pour ne pas dire pécheresse, notamment en écoutant les incantations et airs païens de ces nongermaniques, qu'ils chantaient en toutes circonstances, au travail comme à la maison. [...] De plus, elle avait essayé de mettre sur le papier et d'écrire en toutes lettres ces repoussantes fariboles, qui n'ont ni queue ni tête et sont de pures folies de Satan. ${ }^{27}$ (Kallas 2008, p. 128)

Une autre nouvelle, "La vengeance de la rivière sacrée ", raconte ce qu'il peut en coûter à un étranger de ne pas croire à la réalité des phénomènes surnaturels dont la croyance populaire garde mémoire. Dans le premier passage, le personnage principal, Dörffer, vient de rencontrer un Germano-balte, Gutkind, qui, malgré sa religion, lui semble contaminé par les superstitions locales :

Car il s'avisait de mieux en mieux qu'il était arrivé en étranger dans une terre étrangère, où Satan se manifeste dans le babil des oiseaux innocents et le murmure d'une eau pure, si bien que l'on ne pouvait jamais savoir où se dissimulait le danger, ni où le vif devrait combattre avec le mort, ni l'homme, l'enfant de Dieu, avec la rivière. Heinrich Gutkind lui-même, qui avait reçu le baptême, ne s'était-il pas, depuis à peine une décennie qu'il vivait ici au pays des sorciers, déjà laissé entraîner bien loin dans la superstition ?28 (Kallas 2008, p. 350) 
63 puissance mystérieuse de la rivière dont il essaie de contraindre le cours pour y construire un moulin :

Et Adam Dörffer ne s'étonna pas davantage de cette vision nocturne, mais supposa que cette fille était une des villageoises d'Ilmjärv, car sa vêture était celle des filles du peuple esthonien, et Adam Dörffer savait qu'elles avaient toujours l'habitude de se rendre en secret à la source de la Võhandu pour y maléficier et y porter des offrandes, nonobstant tous les châtiments de l'Église. ${ }^{29}$ (Kallas 2008, p. 351)

Comme on le voit, dans ses récits estoniens, Aino Kallas exploite son intérêt pour le folklore local et en fait le fondement de nouvelles au dispositif narratif bien particulier, où le caractère fermé et sévère du narrateur s'oppose à l'appétit de libertés et à la passion charnelle des héroïnes. L'Estonie, telle qu'elle est décrite, devient le lieu même de cette opposition : le narrateur, esprit chagrin ${ }^{30}$, ne cesse de critiquer l'Estonie, pays envahi de pécheurs et de créatures démoniaques, dans une posture bien différente de celle de l'auteur, dont l'image de l'Estonie était nettement positive.

\section{L'Estonie dans deux romans de Sofi Oksanen}

\section{Les Vaches de Staline : un corps perdu entre deux pays}

Ce roman, le premier de Sofi Oksanen, raconte l'histoire d'Anna, jeune fille souffrant de graves troubles alimentaires (boulimie), que la narratrice semble souvent attribuer au fait qu'Anna est écartelée entre deux pays, l'Estonie par sa mère et la Finlande par son père. Le corps d'Anna devient un véritable symbole de cette difficulté à concilier les deux parts de son identité.

66 L'Estonie est très présente dans le roman, la narratrice revenant constamment sur la façon dont elle perçoit ce pays, qui souvent semble représenter pour elle une idée d'intimité à laquelle ne renvoie pas la Finlande, pays qu'elle ressent presque comme lui ayant été imposé et qui est lié pour elle à l'image d'un père assez médiocre : ainsi, elle exprime son dépit de se voir interdire de parler estonien à la maison (p. 41 de l'édition finlandaise) ; elle manifeste un vif attachement nostalgique envers l'Estonie, mais elle doit dissimuler ce sentiment, même à son compagnon, qui ignore tout de son origine estonienne (p. 192-193). Plusieurs fois, l'Estonie semble liée à des valeurs plus authentiques, plus proches d'elle : ainsi sa grand-mère paternelle, finlandaise, lui paraît bien ordinaire comparée à sa grand-mère estonienne (p. 287-289).

\section{Le corps comme lieu de l'identité}

Le corps de la narratrice, Anna (qui parle régulièrement d'elle à la troisième personne), est le centre thématique du roman et il absorbe toutes les autres problématiques, notamment celle, en apparence secondaire, de l'identité : la question de l'identité est comme lue à travers le prisme des interrogations et des doutes d'Anna sur son corps. Le passage suivant illustre l'importance du corps dans le texte :

Anna ne se fait pas siffler parce que sa mère parle avec un accent. Ni parce qu'on la prend pour une prostituée. Ni parce qu'elle est estonienne, russe, finlandaise, dans le mauvais pays, la mauvaise langue, le mauvais corps. Une fois que son corps a atteint des mensurations adaptées, Anna a pris place au bon endroit, dans le bon 
corps, à la bonne taille, de telle sorte qu'on ne peut que l'approuver. ${ }^{31}$ (Oksanen

2003, traduction de Sébastien Cagnoli, comme les citations suivantes) motif narratif, d'un «Seigneur » qui a pris possession de son corps et qui n'est en fait nul autre qu'une personnification de la boulimie, ainsi valorisée, élevée au rang de divinité au profit de laquelle la narratrice abdique sa liberté.

Ce corps était la demeure de mon Seigneur. Il ne pouvait pas être la mienne, car je n'avais pas d'âme qui aurait besoin d'une demeure charnelle. Mon corps était habité par autre chose, dont le nettoyage et la forme étaient décidés par mon Seigneur, qui lui avait donné une apparence parfaite. Moi-même, je n'aurais pas pu y être. ${ }^{33}$ (Oksanen 2003)

Dans le roman, les deux thématiques de l'identité et du corps sont, de fait, omniprésentes. La narratrice effectue de nombreux voyages en Estonie pour voir sa famille maternelle (l'Estonie est également présente dans les chapitres en analepse où le lecteur assiste à l'évolution des rapports entre les parents de la narratrice) et, de même qu'elle est ballottée entre deux pays, son corps hésite entre plusieurs états, plusieurs poids possibles, plusieurs façons de s'alimenter.

\section{Le corps comme outil de dissimulation} dissimuler ce problème.

La princesse de Finlande attire tellement l'attention qu'il lui devient peu à peu intolérable de passer inaperçue sur une petite place finno-finlandaise, à l'hypermarché ou au feu rouge. C'est pourquoi Anna doit se faire un corps de princesse qui retourne toutes les têtes, mais qui puisse en même temps servir de protection, comme le fait d'être finlandaise derrière la frontière, le genre de protection qui empêche les gens de voir à l'intérieur du corps d'Anna, de voir Anna elle-même. ${ }^{32}$ (Oksanen 2003)

Les troubles alimentaires de la narratrice semblent donc être une conséquence de ses problèmes d'identité: son obsession pour son corps s'explique par la nécessité d'empêcher que l'on s'intéresse à ses hésitations plus fondamentales, à sa perte d'identité due à son appartenance conjointe à deux cultures.

\section{Le corps comme lieu de désappropriation}

La narratrice parle à plusieurs reprises dans le roman, au point d'en faire un important n voit ici le résultat de l'incertitude ontologique que dépeint ce roman : le corps de la narratrice est le berceau de tant de perturbations, d'hésitations, qu'il est en partie conçu par celle-ci comme quelque chose d'étranger, qu'elle ne maîtrise pas et qu'elle a donc abandonné à une entité dépeinte comme extérieure, indépendante de sa volonté.

\section{Purge : isolation et chaos}

Purge raconte l'aventure de Zara, Russe de Vladivostok, d'origine estonienne par sa mère, qui se fait entraîner dans un réseau de prostitution à Berlin et profite d'un voyage en Estonie pour fuir ses ravisseurs/proxénètes et aller retrouver sa grand-tante, Aliide, dont sa grand-mère estonienne lui a parlé. Les deux femmes sont longtemps très 
méfiantes l'une envers l'autre, à cause du poids des rancœurs passées et des secrets de famille liés à l'occupation soviétique.

Du point de vue de la manière dont l'Estonie est évoquée, l'aspect le plus frappant est le sentiment d'isolement qui se dégage des personnages dans les chapitres situés en Estonie. Le plus clair de l'action se passe en effet dans une sorte de huis clos, Zara et Aliide passant leur temps enfermées dans la maison isolée de cette dernière, en bordure de forêt, loin des autres maisons du village. L'isolement est symbolisé de manière encore plus aiguë par une minuscule pièce dissimulée dans la charpente, dans laquelle le beau-frère d'Aliide a passé des années au début de l'occupation soviétique et dans laquelle Zara se cache lorsque ses souteneurs viennent la chercher.

Malgré l'intimité forcée de Zara et Aliide, l'isolement de chacune ne s'efface pas (sauf vers la fin du livre), notamment parce que l'on sent que chacune cache à l'autre beaucoup de détails cruciaux sur son passé : Zara ne dit pas la véritable raison de sa présence et Aliide refuse d'évoquer l'histoire de sa famille.

\section{Les mouches}

76 On trouve dans de nombreuses descriptions de la maison d'Aliide la mention d'essaims de mouches envahissantes qui deviennent peu à peu un véritable symbole, en forme de leitmotiv, des noirs secrets que renferme le passé d'Aliide. Elles apparaissent dès le deuxième chapitre, dont le titre est «La mouche gagne toujours!» (qui contraste avec le titre du premier chapitre, "Vive l'Estonie libre!», les deux phrases faisant référence à une situation de combat) et la première phrase "Aliide Truu fixait la mouche et la mouche lui rendait son regard.» Elles sont également mentionnées dans un passage évoquant la saleté qui caractérise l'Estonie soviétique :

Heureusement, l'enfant grandit et commença à galoper lestement derrière sa mère, y compris à travers les nuées de mouches de l'arrière-cour de la boucherie de Siiri. Parfois les mouches vous entraient dans le nez et les oreilles, parfois on en retrouvait plus tard dans les cheveux [... $]^{34}$ (Oksanen 2010, p. 262).

\section{Le chaos}

77 Même si l'on ne peut nier qu'in fine, le puzzle que constitue la narration éclatée de Purge (alternance, d'un chapitre à l'autre, entre lieux et époques, tous les éléments contribuant au développement de l'intrigue) puisse servir l'effet de suspense, il n'en demeure pas moins que ce roman cultive une esthétique chaotique, qui règne avant tout dans les chapitres concernant l'Estonie. Ainsi, p. 289 et suivantes, on assiste à un périple mouvementé dans la nuit et l'inconnu qui contraste nettement avec les chapitres russe et berlinois, où la ligne narrative est plus dirigée, où les motivations des personnages paraissent limpides. Dans un autre passage esthétiquement révélateur, p. 302 et suivantes, quand Zara essaie de retrouver la ferme de sa grand-tante, l'histoire tragique de la duchesse légendaire Augusta de Koluvere (Augusta de BrunswickWolfenbüttel), cloîtrée en Estonie par ordre de l'impératrice Catherine II, se mêle dans sa tête à ce qu'elle est en train de vivre, formant un mixte qui n'est pas immédiatement débrouillable.

Aide-moi, Augusta, martelait la tête de Zara, et les visages d'Augusta, d'Aliide et de la grand-mère se mêlaient dans son esprit en une seule et même figure et elle n'osait pas regarder à droite ni à gauche, car les arbres de la forêt bougeaient, tendaient leurs membres vers elle. ${ }^{35}$ (Oksanen 2003, p. 303) 


\section{Conclusions}

On a vu que chez chacun de nos deux auteurs, Aino Kallas et Sofi Oksanen, le décor estonien est au centre d'un conflit, le pays ne semble pas choisi au hasard, il est au contraire utilisé en tant que source d'oppositions dramatiques. Chez Kallas, le narrateur vilipende les mœurs estoniennes marquées par la superstition et la soumission au Malin, s'opposant ainsi à des personnages de femmes qui sont au contraire en harmonie avec la nature estonienne et son âme profonde, animale et charnelle si l'on en croit les descriptions de l'auteur; la tension dramatique est constante entre ces deux pôles, ces deux conceptions, entre les idéaux moraux du narrateur et son entourage qu'il perçoit comme immoral. Chez Oksanen, l'Estonie est le lieu par excellence de la crise d'identité, un lieu où il est si facile, tant pour les corps que pour les âmes, d'être écartelé entre la modernité à l'occidentale et le souvenir des désordres soviétiques. ${ }^{36}$

Le contenu dramatique repose par ailleurs sur une impression de chaos qui, chez nos deux auteurs, à des degrés divers, caractérise la façon dont est décrite l'Estonie : chaos politique, chaos moral, chaos dans les relations entre les personnages ou dans l'esprit du narrateur.

Il est difficile, dans le cas d'oksanen, de savoir si ce chaos procède d'une volonté de transcrire une impression réelle de l'Estonie (due à l'expérience personnelle de l'auteur), ou si c'est un pur artifice narratif, une manière de rendre les oppositions dramatiques plus intéressantes en les situant dans un lieu qui les reflète. Chez Kallas en revanche, il est manifeste qu'il s'agit d'une ruse narrative, car on sait que l'auteur avait une conception autrement paisible et affectueuse de son pays d'élection. La vision négative qui émane des récits estoniens de Kallas a quelque chose d'ironique, qui vise davantage les narrateurs, à l'esprit obtus et marqué par des idéologies intolérantes, que le pays qui constitue le décor des textes.

\section{BIBLIOGRAPHIE}

Kallas Aino, 1978, Elämäni päiväkirjat, Otava, Helsinki (deux volumes).

Kallas Aino, 2008, Valitut teokset, Otava, Helsinki.

Minaudier Jean-Pierre, 2007, Histoire de l'Estonie et de la nation estonienne, L'Harmattan, Paris.

Oksanen Sofi, 2003, Stalinin lehmät, WSOY, Helsinki.

Oksanen Sofi, 2008, Puhdistus, WSOY, Helsinki.

Oksanen Sofi, 2010, Purge, Stock, Paris, traduction de Sébastien Cagnoli.

RAUSMAA Heikki, 2007, Tuglaksen tuli palava, SKS, Helsinki

Études finno-ougriennes, 46 | 2014 


\section{NOTES}

1. La source principale de cet éclairage historique est Rausmaa 2007.

2. Yrjö Sakari Yrjö-Koskinen (1830-1903), sénateur, historien, successeur de Snellman à la tête du parti fennomane.

3. J. V. Jannsen (1819-1990), figure de proue de l'éveil national estonien, fondateur en 1857 du Pärnu Postimees, hebdomadaire qui joua un rôle important dans l'éveil national.

4. Carl Gustaf Swan (1839-1916), professeur et journaliste finlandais.

5. Carl Robert Jakobson (1841-1882), journaliste estonien, figure importante de l'éveil national, fondateur du journal Sakala, où il revendiquait des réformes en faveur des Estoniens.

6. Cf. Minaudier 2007, p. 198-202.

7. Mouvement politique finlandais à tendances fascistes, actif entre 1929 et 1932.

8. Tentative de coup d'État, en mars 1932, par les militants anticommunistes du mouvement de Lapua.

9. La Ligue voyait le mouvement de Lapua comme un modèle, mais était bien moins violente et ne se référait pas au nazisme. Nous renvoyons à Minaudier 2007, p. 267-271, pour cet épisode.

10. Konstantin Päts (1874-1956) fut plusieurs fois chef de l'État estonien entre 1921 et 1940.

11. SKS : Suomalainen Kirjallisuuden Seura, Société de Littérature finnoise.

12. Voir Rausmaa 2007 et Minaudier 2007 pour plus de précisions sur ce contexte historique.

13. Kerran olin suuremmassa seurassa, mutta palasin sieltä melkein itku kurkussa, niin oli ollut ikävä. Naiset kaikki pikkumaisia, ilman intressejä, kiinni pienessä, ahtaassa näköpiirissään. Miehet ovat ainakin isänmaallisia, pitävät kiinni omasta kielestään ja kansallisuudestaan, naiset eivät välitä siitäkään. Palvelijat ja talous, lisäksi perheintrigit - siinä heidän maailmansa.

14. Virolaisuus on käännekohdassaan, toisella puolella Scyllana saksalaisuus, toisella venäläinen Charybdis. Luoviiko se onnellisesti läpi? Niin, jos naiset heräävät!

Nämä balttilaiset naiset, joitten ajatuspiiri on niin kaita ja ahdas, jotka eivät osaa keskustella, eivät innostua, eivät ajatella! [...]

Olen saanut 'virolaisen herätykseni' aivan negatiivista tietä, Rudolf Kallaksen rouvan ja tytärten kautta, sentähden, että he ovat virolaisuudelle aivan kylmiä ja välinpitämättömiä, vieläpä halveksien kohtelevat kaikkia virolaisia pyrintöjä. [...]

Nuorempi tytär, Grete, kaunis, runollinen, hiukan pintapuolinen koulutyttö:

«Mitä minä voin sille, että rakastan saksalaisia monta vertaa enemmän kuin virolaisia ? Viron kansahan on niin kauhean typerää !

« Todella - isäsikö myös?

« Oh - eihän isä kuulu peupleen!

Tytöltä ei saa viron sanaa suusta, vastaa itsepäisesti saksaksi, lukee suurella vaivalla viroa sisältä ja sanoo ymmärtävänsä sitä vain osaksi. [...]

Aksel, 11-vuotias pikku poika, ei omain sanainsa mukaan koskaan ole pitänyt käsissään vironkielistä kirjaa. Hämmästyi suuresti kuullessaan, että hänen isänsä on kuulunut 
Eesti Üliõpilaste Seltsiin [...] ja sanoi itse tahtovansa kuulua saksalaiseen korporatsiooniin.

15. Teidän arvostelussanne oli eräs kohta, jossa kosketitte oikeastaan sangen arkaa paikkaa minussa. Tarkoitan 'juurettomuuttani' ja suhdettani virolaisten olojen ja olojen sielun ymmärtämykseen tai ei-ymmärtämykseen.

16. Suomessa olisin tuskin siinäkään määrässä voinut eläytyä todellisuuteen kuin Virossa, jossa olojen outous avasi silmäni (Kallas 1978, p. 407).

17. Erehdykseni on siinä [...] että tahdoin käsitellä virolaisia probleemoja. Siihen ei minun sisäinen näkemykseni tietysti riitä. Se on tehtävä, joka jää virolaisille kirjailijoille. Mutta ymmärrättehän Te erotuksen, kun sanon, että minulla häilyy mielessä jotain tämäntapaista: käsitellä yleisinhimillisiä probleemoja, mutta virolaisin henkilöin. Ehkä tämä ei ole kyllin selvästi sanottu, mutta en sitä tänä hetkenä osaa paremmin sanoa. Sillä mistä ottaisin tulevaisuudessa henkilöni - en ainakaan Suomesta ! [...]

Niin että jos nyt voisin vapaasti valita, en enää pyytäisi itselleni juuria en Suomessa enkä Virossa, vaan siinä suuressa ihmisyydessä, johonka me jokainen voimme juurtua ja siinä kasvaa.

18. Rakastan Suomea, mutta Viron kohtalo on enemmän sydämelläni.

19. Viisitoista vuotta olen nyt asunut Virosssa, kahdeksantoista saman kansan ajatuspiirissä, olen ollut aina vieras, aina ulkopuolella - tähän saakka! [...] Suomi on minulle hajonnut vain yksityisiksi ihmisiksi, kollektiivisesti katsottuna on Viro nyt kotimaani.

20. « Satujen saari », Kallas 1978 p. 564

21. Saarenmaa otti minut vastaan lämpimällä suviyöllä. Ajoimmme hämärissä, koivut tuoksuivat, Sulev lepäsi sylissäni, täysikuu paistoi halavien läpi. Ajattelin, kuinka kaunis, ihana poika minulla on. Ajattelin kiitollisuudella Saarenmaata, koska tunnen täällä - ja ainoastaan täällä - itseni kotiutuneeksi.

22. Millaista Tartossa oli - ahdastako? hän kysyi. - Ahdasta, kuten tavallista, vastasin.

23. Mikä ihana paikka, tämä Pärnu - yksilö ikävien tusinakaupunkien joukossa, täynnä ihastuttavia yksityiskohtia ja kokonaisuuden viehätystä. [...] Meri, laivat - ikäänkuin silta kaukaisiin maailmoihin; milloinkaan ei merikaupungissa voisi yllättää sydäntä puristava ahtaus, kuten sisämaan pikkukaupungissa (Kallas 1978, II, p. 108).

24. Mutta niinä aikoina [...] kasvoi Liivinmaalla hyvinvointi ja rikkaus, mutta myös Saatanan kylvöstä kaikkinainen rikkaruoho, kuin on prameus ja hekuma sekä hallitsijain että alamaisten seassa. Sillä porvarit ja myöskin epäsaksalaiset talonpojat ovat herrojen esimerkin jälkeen tehneet, niin ettei ole toista kansaa koko kristikunnassa joka olisi elänyt tainkaltaisessa haureudessa ja sodomalaisten sekä epikurolaisten tavalla, itsekukin etsein hänen autuuttansa vain maallisessa ja synnillisessä ilonpidossa.

25. Mutta minun tullessani Tallinnaan oli Viron- sekä Liivinmaalla käyty sotia lähes seitsemänkymmentä ajastaikaa lakkaamatta, ja koko maa oli niinkuin olisivat Pyhän Ilmestyskirjan orhit sen kavioillansa kuopineet. Kirkot oli poltettu elikkä muuten häväisty [...] Eipä ollut usein papeissakaan muuta parempata pappeutta kuin musta kaapunsa. [...] Vain Saatana, se Helvetin Vanha Nuottamies, kulki ympärinsä ja veti verkkojansa veden sameudessa, mesi mielessä. Tosin noitaroviot roihusivat pitkin maata ja polttivat ohdakkeita ja rikkaruohoja, niin että käry suitsusi, mutta uusia 
hurmanhenkiä tuli kuin Helvetin säkin suusta, sillä Perkele näkyi päättäneen, ettei Vironmaan pitänyt autuaaksi tuleman.

26. Mutta nämä julkiat susilaumat olivat vain niinkuin Helvetin esijoukot, jotka se Rietas Henki, kuin ihmisen kadotuksen jälkeen aina ankarasti seisoo, edellänsä lähetti.Sillä ei kauvan senperästä, kun luonnolliset sudet näin riettaiksi riehastuivat, niin ettei missän tainnut heidän elkeiltänsä turvassa olla, niin jo alkoivat ihmisenlapsetkin susina juosta ja sudentekoja tehdä, niinkuin olisi häijy Daimoni heihin mennyt. [...]Niin myös Hiidenmaalla usiat Herran laumasta lankesivat tähän uuteen loukkuun, jonka Saatana oli laittanut.

27. Myöskin on hän alkanut viettää aikaansa niin turhanpäiväisellä, etten sanoisi synnillisellä tavalla, kuin kuuntelemalla näitten epäsaksalaisten pakanallisia loitsuja ja viisuja, joita he työssä ollessaan kuin myös kotonansa jokaisessa tilaisuudessa laulavat. [...] Vieläpä hän on yrittänyt panna paperille ja ylöskirjoittaa puustaveilla näitä inhoittavia loruja, joilla ei ole päätä eikä perää, ja jotka ovat Saatanan villitystä alusta loppuun. (128)

28. Sillä yhä selkiämmin hän tiesi tulleensa muukalaisena outoon maahan, kussa Saatana ilmaisee itsensä viattomain lintujen liverryksessä ja syyttömän veden solinassa, niin ettei tiedä, mistä vaara kulloinkin väijyy, ja kussa elollisen on kamppailtava elottoman, ja ihmisen, Jumalan lapsen, yhden joen kanssa. Eiköstä ollut Heinrich Gutkind, hyvä kristitty, vain vuosikymmenen täällä velhoin maassa viivyttyänsä ennättänyt perin syvälle harhauskoon hairahtaa?

29. Niin Adam Dörffer ei tätä yöllistä näkyä sen enempätä hämmästellyt, vaan arveli tytön olevan Ilmjärven kyläläisiä, sillä hänen vaatepartensa oli maarahvaan tyttärien, ja Adam Dörffer tiesi heidän yhä salaa Võhandun lähteellä taikomassa ja uhria tuomassa käyvän, kaikista kirkon vakavista nuhteista välittämättä.

30. Esprit chagrin et donc généralement déplaisant aux yeux du lecteur, mais pas de manière systématique : le pasteur de Reigi, par exemple, est certes coupable envers son épouse, mais l'auteur met également en avant sa nature de victime, et le personnage ne laisse pas d'attendrir.

31. Annalle ei vihelletä sen takia, että hänen äitinsä puhuu korostuksella. [...] Ei siksi, että hänen kuvitellaan olevan prostituoitu [...]. Ei siksi, että hän on virolainen, venäläinen, suomalainen, väärässä maassa väärässä kielessä väärässä ruumiissa. Saatuaan ruumiinsa mitat kohdalleen Anna on solahtanut oikeaan paikkaansa, oikeaan ruumiiseensa, oikeaan kokoonsa ja tullut juuri sillä tavalla hyväksytyksi kuin on tarpeen. (232)

32. Suomen prinsessa [Virossa] saa huomiota niin paljon, että näkymättömäksi muuttumisesta sysisuomalaisen toripahasen kupeessa tai automarketissa tai liikennevaloissa tulee vähitellen aivan sietämätöntä. Siksi Annan pitää prinsessoida ruumiinsa sellaiseksi, että se kääntää kaikki päät, mutta toimii samalla suojana, kuten suomalaisuus rajan takana, samanlaisena suojana, joka estää näkemästä Annan ruumiin sisälle, Annaa itseään. (232) Cf. également p. 336.

33. Tämä ruumis oli Herrani koti. Minun ruumiini ei voinut olla kotini, sillä minulla ei ollut sielua, joka olisi tarvinnut lihallisen kodin. Minun ruumistani asui joku muu, sen siivouksesta ja muodosta päätti Herrani, joka oli tehnyt siitä täydellisen näköisen. Minusta itsestäni ei siihen olisi ollut. (316) 
34. Onneksi lapsi kasvoi ja alkoi pinkoa sujuvasti äitinsä perässä myös Siirin lihakaupan takapihan kärpäsparven lävitse. Joskus kärpäsiä meni nenään ja korviin, joskus niitä löytyi myöhemmin tukasta [...]

Sur les mouches, voir également Oksanen 2010, p. 319.

35. Auta, Augusta, Zaran päässä takoi, ja Augustan, Aliiden ja isoäidin kasvot sekoittuivat hänen mielessään yhdeksi ja samaksi eikä hän uskaltanut uskoa oikealle eikä vasemmalle, sillä metsän puut liikkuivat, niiden raajat ojentuivat häntä kohti.

36. De façon plus anecdotique, on peut citer un autre point commun, le motif dramatique - courant dans la littérature estonienne, symbole de désillusion, d'attente trompée - du " bateau blanc ", le valge laev, qui apparaît fugitivement dans Les Vaches de Staline, d'Oksanen, p. 274, et est un ressort essentiel d'une nouvelle de Kallas, «Le bateau blanc de Lasnamäe ».

\section{RÉSUMÉS}

Cet article, après avoir évoqué l'histoire des relations culturelles finno-estoniennes, examine la façon dont est évoquée l'Estonie chez deux auteurs finlandais dans l'œuvre desquels ce pays joue un rôle de premier plan : Aino Kallas, dans la première moitié $\mathrm{du} \mathrm{xx}^{\mathrm{e}}$ siècle, a consacré à l'Estonie plusieurs de ses nouvelles les plus importantes, et, en particulier, son cycle de l'Éros assassin, tandis que Sofi Oksanen s'est spécialisée dans les romans où l'histoire récente de l'Estonie sert de toile de fond à des histoires à suspens et à des questionnements identitaires. Notre analyse montre l'importance de la peinture de l'Estonie dans la construction dramatique des textes considérés : la vision négative qu'ont de l'Estonie les narrateurs mis en scène par Aino Kallas est au cœur d'un système d'opposition entre les personnages, et chez Sofi Oksanen, les personnages sont largement déterminés par leur identité estonienne, qui est toujours source de conflits, pour des raisons psychologiques ou historiques.

This article, after summarising the history of Finnish-Estonian cultural relations, deals with the way Estonia is shown in the texts of two Finnish writers in whose works Estonia plays a foremost role. In the first half of the 20th century Aino Kallas dedicated some of her most important short stories to Estonian themes, particularly her cycle about "Eros the Slayer", while Sofi Oksanen has specialised in novels where the recent history of Estonia appears as a background for suspense stories and identity questioning. Our analysis shows the importance of the depiction of Estonia in the dramatic construction of the texts considered: the negative considerations displayed by Aino Kallas' narrators are the core of an opposition system between the characters, and in Sofi Oksanen's books, the characters are largely defined through their Estonian identity, which is always a source of conflict, for either psychological or historic reasons.

Selles artiklis võetakse kokku Soome-Eesti kultuurisuhete ajalugu ning vaadeldakse seejärel, mil viisil on Eestit kujutatud kahe Soome kirjaniku teoses, milles Eesti on esiplaanil. 20. sajandi esimesel poolel pühendas Aino Kallas Eestiga seotud teemadele mõned oma tähtsamatest lühijuttudest, eriti „Surmava Erose“ tsüklist. Sofi Oksanen aga kirjutab romaane, milles tegelaste identiteedid on seotud Eesti lähiajaloo sündmustega. Artikli analüütilises osas näidatakse Eesti tähtsust uuritavate tekstide dramaatilises ülesehituses: Aino Kallase jutustajate negatiivsed 
hinnangud on tegelastevahelise vastandumise keskmes. Sofi Oksaneni raamatutes aga määratleb suure osa tegelastest nende Eesti identiteet, mis on alati, kas psühholoogilistel või ajaloolistel põhjustel konflikti allikaks.

\section{INDEX}

Index chronologique : XXe siècle (début), XXIe siècle (début), XXe siècle, XXIe siècle Mots-clés : AKS Société universitaire carélienne, corps, Guerre d'hiver, échanges culturels, Lettre des quarante, éveil national, relations finno-estoniennes, Ligue des anciens combattants de la guerre d'indépendance, Mouvement de Lapua, Révolte de Mäntsälä, Sakala, SKS [Suomalaisen kirjallisuuden seura], SNS [Suomi-Neuvostoliitto seura], Union estonienne des écrivains, Société Tuglas [Tuglas-seura], Pärnu Postimees

disciplines allemand, estonien, finnois, langues fenniques

Index géographique : Allemagne, Berlin, Estonie, Finlande, Helsinki, Hongrie, Jyväskylä,

Laajasalo, Luxembourg, Moscou, Pärnu, Pologne, Prague, Fédération de Russie, Saaremaa, Sibérie, Sõrv, Suède, Tallinn, Tartu, Vladivostock

Thèmes : littérature comparée

Keywords : Body, Cultural Exchange, Finnish-Estonian relations, national awakening, beginning of the 20th century, beginning of the 21st century, Estonian, Finnish, Finnic languages, German, Estonians, Finno-Ugric peoples, Finns, Germans, Russians, Swedes

nomsmotscles Allemands, Estoniens, Finlandais, Russes, Suédois 